\title{
Fibrosi cistica: come cambierà la malattia con i nuovi modulatori della proteina CFTR?
}

\author{
Cesare Braggion \\ Direzione scientifica, Fondazione Ricerca Fibrosi Cistica
}

In quest'ultimo decennio la ricerca ha portato alla ribalta nuovi farmaci per la cura della fibrosi cistica (FC), i cosiddetti modulatori della proteina CFTR (Cystic Fibrosis Transmembrane Conductance Regulator). Questa proteina, prodotto del gene CFTR, ha funzioni di canale per il trasporto di cloro e bicarbonato, inoltre inibisce il trasporto del sodio a livello della membrana apicale delle cellule e perciò regola il $\mathrm{pH}$ e il trasporto di acqua sulla superficie delle cellule. Le alterazioni della proteina CFTR, che possono interessare la sua funzione (difetto di apertura del canale, difetto di conduttanza, scarsa quantità sulla membrana cellulare, instabilità sulla membrana cellulare e inattivazione) o la sua presenza sulla membrana cellulare (difetto di sintesi, difetto di conformazione e di posizionamento) sono diverse in base alle diverse mutazioni del gene [1]. Dall'alterazione della proteina CFTR dipendono tutti i sintomi della FC con un interessamento multiorgano e con caratteristiche di evolutività, specie per l'interessamento polmonare. La variabilità fenotipica è peraltro ampia ed è spiegata dal genotipo, ma anche da altri geni "modificatori", dall'ambiente, da alcune caratteristiche cliniche e dal contesto sociale e dell'organizzazione del sistema sanitario.

I modulatori della proteina CFTR hanno dimostrato nei trial di fase 3 di incidere sulla proteina CFTR difettosa ma anche di essere efficaci sui sintomi e sicuri nel loro uso [2]. Il cloro sudorale, gold standard diagnostico per la malattia, è stato utilizzato come biomarcatore: la sua riduzione più o meno marcata indica un ripristino del trasporto del cloro e perciò un effetto diretto sulla proteina CFTR. Il miglioramento dei parametri della spirometria, la riduzione del numero di esacerbazioni polmonari, la riduzione dei sintomi respiratori, il miglioramento dello stato nutrizionale rappresentano benefici diretti per le persone con fibrosi cistica (FC), per le quali questi nuovi farmaci sono stati indicati (Figure 1-2).
La mossa vincente per l'identificazione di questi farmaci è stata l'applicazione del metodo dello screening ad alta efficienza (high-throughput screening) di grandi librerie di farmaci [3]. Questo consi-

A) Aumento medio del $\mathrm{FEV}_{1}$ in punti di percentuale predetta rispetto al valore misurato con il placebo

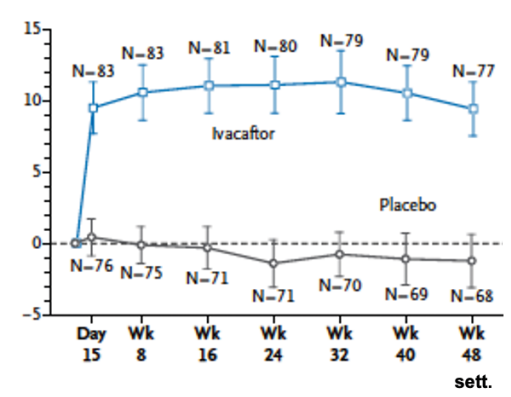

ste in piastre di cellule epiteliali di tiroide di ratto Fisher, il cui gene CFTR è stato modificato per contenere specifiche mutazioni: queste cellule possono esprimere sia la proteina CFTR sia una proteina fluo-

B) Riduzione media in $\mathrm{mmol} / \mathrm{L}$ del cloro nel sudore a partire dal valore in terapia con il placebo (valore patologico basale)

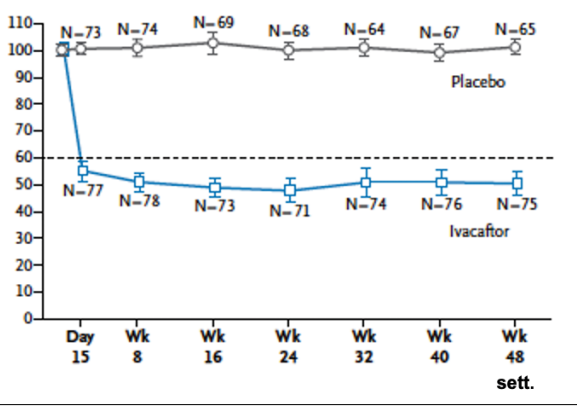

Figura 1. Effetti principali del Kalydeco ${ }^{\circledR}$ nelle persone con una mutazione di gating (G551D), riportati dal trial clinico di fase 3 [4]. In A è riportato l'aumento medio del FEV, (volume espiratorio forzato al primo secondo), parametro misurato con la spirometria e considerato per la fibrosi cistica l'end point

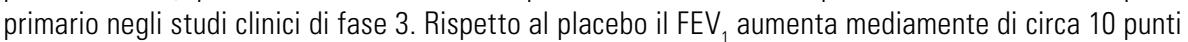
di percentuale predetta, che corrisponde a un aumento relativo del $17 \%$ rispetto al valore basale. In B è riportata la riduzione media in $\mathrm{mmol} / \mathrm{L}$ del cloro sudorale, rispetto al valore registrato durante la terapia con placebo: il valore del cloro scende sotto il limite patologico e perciò diagnostico di malattia, che corrisponde a $60 \mathrm{mmol} / \mathrm{L}$.

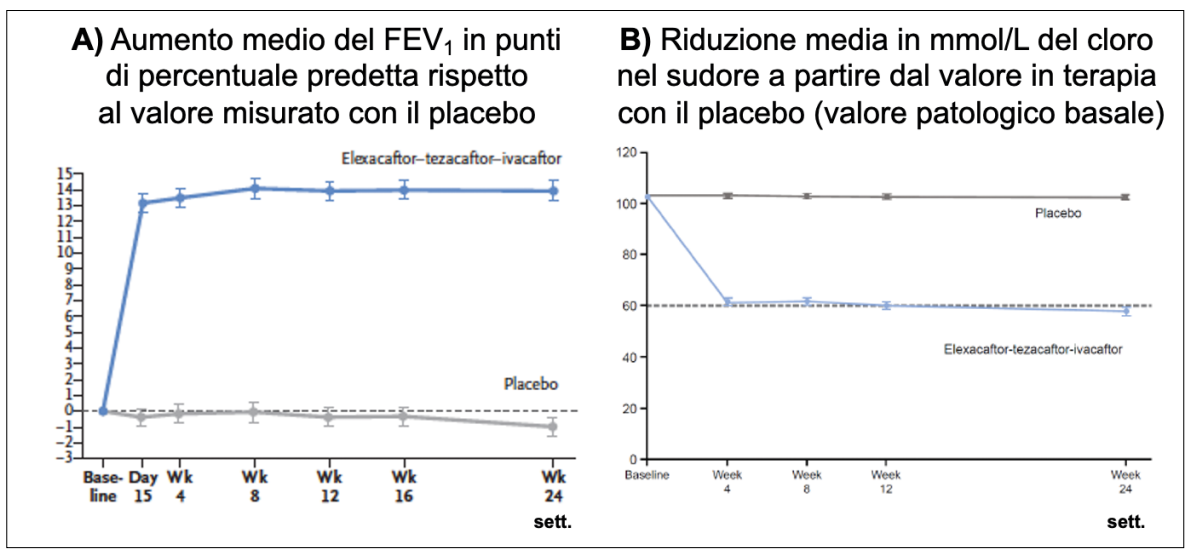

Figura 2. Effetti principali del Kaftrio ${ }^{\circledR}$ nelle persone con una mutazione F508del e una mutazione a funzione minima nel secondo allele, riportati dal trial clinico di fase 3 [6]. In A è riportato l'aumento medio del $\mathrm{FEV}_{1}$ (volume espiratorio forzato al primo secondo), parametro misurato con la spirometria e considerato per la fibrosi cistica il principale end point negli studi clinici di fase 3. Rispetto al placebo il $\mathrm{FEV}_{1}$ aumenta mediamente di circa 14 punti di percentuale predetta. In B è riportata la riduzione media di circa $42 \mathrm{mmol} / \mathrm{L}$ del cloro sudorale, rispetto al valore registrato durante la terapia con placebo: il valore del cloro arriva intorno al limite patologico e perciò diagnostico di malattia, che corrisponde a $60 \mathrm{mmol} / \mathrm{L}$. 
rescente, che è sensibile al passaggio del cloro. Le cellule vengono in contatto con decine di migliaia di farmaci noti: nel caso che qualcuno di questi sia in grado di attivare la proteina CFTR, il flusso di cloro attiva la proteina fluorescente che rappresenta il segnale di attività del composto. Quest'ultimo viene poi studiato nella sua funzione di attivazione della proteina CFTR e del trasporto del cloro in un altro modello cellulare, quello delle cellule umane bronchiali, prelevate da polmoni di persone con FC nel contesto del trapianto polmonare e perciò con un ben definito genotipo. Se confermato efficace, il composto e gli analoghi chimici vengono studiati nel loro profilo di farmacocinetica e sicurezza nell'animale. Se anche questo step viene superato possono iniziare gli studi nella persona con FC. Tutto ciò avviene senza la conoscenza dei meccanismi d'azione dei farmaci e senza gli studi preliminari di efficacia e sicurezza nei modelli animali con FC.

A posteriori possiamo dire che la strategia dell'high-throughput screening è stata vincente e relativamente rapida, poiché in circa vent'anni disponiamo di almeno due farmaci, il Kalydeco ${ }^{\circledR}$ e il Kaftrio ${ }^{\circledR}$, incisivi sulla malattia, poiché in grado di ridurre decisamente il cloro sudorale e di migliorare alcuni sintomi e segni caratteristici della FC (Figure 1-2). Il primo è un potenziatore, l'ivacaftor, che è stato dimostrato agire direttamente sull'apertura della proteina-canale CFTR (gating) nel caso delle mutazioni, come la G551D, che sono caratterizzate dalla presenza in membrana della proteina, che ha però questo difetto di funzione [4-5]. Il secondo corrisponde alla combinazione dello stesso potenziatore ivacaftor e di due correttori, l'elexacaftor e il tezacaftor, che si sono dimostrati incisivi sul difetto di CFTR, prodotto dalla mutazione F508del. Il difetto prodotto da questa mutazione è sia di conformazione della proteina e trasporto verso la membrana apicale (traffiking), sia di apertura del canale (gating) [6-7]. I risultati dei trial clinici di fase 3 per i due farmaci sono stati pubblicati, rispettivamente, nel 2011 e 2019. Le Figure 1-2 illustrano i risultati principali ottenuti dai due farmaci. Prima del Kaftrio, sono stati autorizzati e resi disponibili per la prescrizione da parte dei centri FC, l'Orkambi ${ }^{\circledR}$ (combinazione del potenziatore ivacaftor e del correttore lumacaftor) e il Symkevi ${ }^{\circledR}$ (combinazione del potenziatore ivacaftor e del correttore tezacaftor): entrambi questi farmaci sono stati testati e approvati per chi aveva la doppia mutazione F508del, ma i risultati clinici dimostrati nei trial di fase 3 sono stati ben inferiori a quelli ottenuti con il
Kalydeco [8-9]. Ciò ha convinto della necessità di identificare modulatori più incisivi sui difetti della mutazione F508del: la tripla combinazione di modulatori, il Kaftrio, si è dimostrata efficace non solo negli omozigoti F508del ma anche in coloro che sono eterozigoti per la mutazione F508del, avendo sul secondo allele una mutazione diversa. I trial clinici hanno consentito di allargare l'indicazione del Symkevi a coloro che hanno una mutazione F508del e una mutazione a funzione residua sul secondo allele [10]. Queste ultime mutazioni si accompagnano a normale funzione digestiva e a una malattia polmonare più lieve rispetto a coloro che hanno una o due mutazioni F508del.

Mentre i primi trial clinici e le successive autorizzazioni all'immissione in commercio hanno riguardato adulti e adolescenti con FC (età superiore o uguale ai 12 anni), tutti i modulatori sono stati testati progressivamente anche in età pediatrica. Le ultime autorizzazioni di AIFA all'immissione in commercio a spese del SSN, che risalgono al 5 luglio 2021, hanno riconsiderato tutti i modulatori in commercio:

1 il Kalydeco per le mutazioni di gating a partire da un età di 12 mesi e la mutazione R117H solo per gli adulti: ciò riguarda circa 180 persone con FC, pari al 3,3\% rispetto alla popolazione italiana, secondo i dati del 2018 riportati dal Registro italiano fibrosi cistica;

2 l'Orkambi per la doppia mutazione F508del a partire da un'età di 2 anni: ciò riguarda circa 1.100 persone con FC, pari al 20,2\%;

3 il Symkevi per la doppia mutazione F508del e gli eterozigoti per F508del e per una di 14 tra le mutazioni a funzione residua, a partire da un'età uguale o superiore ai 12 anni: ciò riguarda circa 1.170 persone, pari al 21,3\%;

4 il Kaftrio per la doppia mutazione F508del e gli eterozigoti per F508del e una mutazione a funzione minima sul secondo allele a partire da un'età uguale o superiore ai 12 anni: ciò riguarda circa 1750 persone, pari al 31,9\% [11].

Le mutazioni a funzione minima sono circa 200: sono caratterizzate negli studi in vitro dall'assenza della proteina CFTR sulla membrana cellulare, da un trasporto del cloro CFTR-dipendente inferiore al 10\% rispetto a quello normale e da una variazione assente o minima di quest'ultimo dopo la somministrazione di ivacaftor, tezacaftor e ivacaftor-tezacaftor [6]. Tenendo conto che alcuni di questi farmaci, autorizzati recentemente da AIFA, sono prescrivibili per gli stessi pazienti e considerando le fasce di età considerate, attual- mente la somministrazione dei modulatori della proteina CFTR riguarda in Italia circa 2.600 persone con FC, pari al 47,5\%. All'orizzonte c'è l'allargamento dell'indicazione del Kaftrio a tutti coloro che hanno una mutazione F508del e qualsiasi altra mutazione nel secondo allele: questa indicazione è stata già autorizzata da EMA [12]. Ciò porterebbe l'indicazione del farmaco da 1.750 a 2.700 persone $(49,3 \%)$. Si attende un pronunciamento dell'agenzia europea anche per l'indicazione del farmaco a partire da un'età di 6 anni [13].

Se consideriamo i due modulatori di CFTR più efficaci, Kalydeco e Kaftrio, e la loro indicazione che comprende, rispettivamente, coloro che hanno almeno una mutazione di gating/R117H o F508del su un allele e qualsiasi altra mutazione sul secondo allele e l'appartenenza a qualsiasi fascia di età, la popolazione che può assumere uno dei due farmaci interessa circa 3.800 persone, pari al 69,9\% della popolazione italiana con FC [11]. Il risvolto negativo è rappresentato dal fatto che il 30,1\% (1.653) delle persone FC non può disporre attualmente di un farmaco modulatore della proteina CFTR. Questo gruppo, che comprende soggetti che non hanno né una mutazione di gating/R117H, né F508del su alcun allele è rappresentato da:

- coloro che hanno almeno una mutazione a funzione residua;

- coloro che hanno almeno una mutazione di "stop", caratterizzata dalla presenza di codoni, cosiddetti di stop, che impediscono all'RNA messaggero di sintetizzare una proteina CFTR completa;

- coloro che hanno una mutazione a funzione minima non correggibile, come la mutazione N1303K, la seconda mutazione per frequenza in Italia, o una mutazione rara, della quale non è nota la funzione e perciò la potenziale terapia con modulatori CFTR.

Se consideriamo la frequenza delle diverse mutazioni, la situazione italiana è ben diversa rispetto ai Paesi del Nord Europa e degli Stati Uniti. La mutazione F508del rimane la mutazione più frequente anche in Italia. Se, per esempio, confrontiamo il nostro Paese con il Regno Unito, gli omozigoti per la mutazione F508del sono rispettivamente il $21 \%$ e il $44 \%$, gli eterozigoti per la mutazione F508del sono rispettivamente il $47 \%$ e $46 \%$. In Italia, rispetto agli altri Paesi, sono più frequenti le mutazioni rare e quelle a funzione residua.

Merita soffermarci sul costo di questi farmaci. Lultima contrattazione di AIFA con Vertex Pharmaceuticals, azienda pro- 
duttrice dei modulatori, ha compreso non solo il farmaco Kaftrio, ma anche una riduzione dell'età di indicazione dell'Orkambi e di Kaydeco e l'autorizzazione al commercio di Symkevi. La proposta di una contrattazione allargata è venuta $d a$ Vertex Pharmaceuticals: questa strategia è stata portata avanti dall'azienda in tutti i Paesi europei. Solo i farmaci Kaftrio e Kalydeco sono stati considerati innovativi e perciò il loro costo è a carico del Fondo AIFA per i farmaci innovativi [14]. Il costo ex-factory del Kaftrio è di circa 244.000 euro anno/paziente. Se consideriamo le 1.750 persone, a cui il farmaco è destinato, il suo costo è pari a 427 milioni/ anno di euro. La contrattazione tra AIFA e l'azienda farmaceutica ha portato, come è avvenuto per altri farmaci, a uno sconto sul prezzo ex-factory per le strutture sanitarie pubbliche e a un "tetto" complessivo di spesa per tutti i farmaci prodotti dall'azienda. Occorre sottolineare che al momento Vertex Pharmaceuticals ha il monopolio dei farmaci modulatori di CFTR. Nonostante l'avanzamento nella cura della FC dopo l'inserimento dei modulatori di CFTR rimangono molte questioni aperte, a cui la ricerca deve dare una risposta:

- si confermerà il profilo di efficacia e sicurezza dei nuovi farmaci nella real life e per una durata di somministrazione maggiore di 2 anni?

- quale nuova terapia si può proporre per un terzo delle persone con FC che allo stato attuale non dispongono di un farmaco incisivo sul difetto di base?

- potranno essere proposti nuovi modulatori con un profilo di efficacia e sicu- rezza migliore degli attuali, favorendo la concorrenza e perciò portando a una riduzione dei costi per il SSN?

- sarà applicabile per la prescrizione lo studio dell'efficacia dei farmaci modulatori, dimostrabile nelle cellule nasali o rettali, prelevate dal singolo individuo, specie in coloro che hanno mutazioni rare o non responsive a uno o più farmaci disponibili (theratyping)?

All'orizzonte occorre anche considerare le nuove sperimentazioni in vitro sulla possibilità di editing di DNA e RNA, cioè di modificare con molecole "taglia $\mathrm{e}$ incolla" il DNA e l'RNA inserendovi l'informazione normale: ciò consentirebbe di agire indipendentemente dalle mutazioni del gene, ma molti sono ancora i problemi da risolvere. L'orizzonte della ricerca resta perciò ancora ampio!

\section{$\bowtie$ cesarebraggion.133@gmail.com}

1. Bell SC, Mall MA, Gutierrez H, et al. The future of cystic fibrosis care: a global perspective. Lancet Respir Med. 2020 Jan;8(1):65-124. 2. Mayer-Hamblett N, Boyle M, Van Devanters D. Advancing clinical development pathways for new CFTR modulators in cystic fibrosis. Thorax. 2016 May;71(5):454-461.

3. Ma T, Vetrivel L, Yang H, et al. High-affinity activators of cystic fibrosis transmembrane conductance regulator (CFTR) chloride conductance identified by high-throughput screening. J Biol Chem. 2002 Oct 4;277(40):37235-37241.

4. Ramsey BW, Davies J, McElvaney NG, et al. A CFTR potentiator in patients with cystic fibrosis and the G551D mutation. N Engl J Med. 2011 Nov 3;365(18):1663-1672.
5. De Boeck K, Munck A, Walker S, et al. Efficacy and safety of ivacaftor in patients with cystic fibrosis and a non-G551D gating mutation. J Cyst Fibros. 2014 Dec;13(6):674-80.

6. Middleton PG, Mall MA, Dřevínek P, et al. Elexacaftor-Tezacaftor-Ivacaftor for Cystic Fibrosis with a Single Phe508del Allele. N Eng1 J Med. 2019 Nov 7;381(19):1809-1819.

7. Heijerman HGM, McKone EF, Downey DG, et al. Efficacy and safety of the elexacaftor plus tezacaftor plus ivacaftor combination regimen in people with cystic fibrosis homozygous for the F508del mutation: a double-blind, randomised, phase 3 trial. Lancet. 2019 Nov 23;394(10212):1940-1948.

8. Wainwright CE, Elborn JS, Ramsey BW, et al. Lumacaftor-Ivacaftor in Patients with Cystic Fibrosis Homozygous for Phe508del CFTR. N EnglJ Med. 2015 Jul 16;373(3):220231.

9. Taylor-Cousar JL, Munck A, McKone EF, et al. Tezacaftor-Ivacaftor in Patients with Cystic Fibrosis Homozygous for Phe508del. N Engl J Med. 2017 Nov 23;377(21):2013-2023. 10. Rowe SM, Daines C, Ringhausen FC, et al. Tezacaftor-Ivacaftor in Residual-Function Heterozygotes with Cystic Fibrosis. N Engl J Med. 2017 Nov 23;377(21):2024-2035.

11. Campagna G, Amato A, Majo F, et al. Registro Italiano Fibrosi Cistica. Report 20172018. Epidemiol Prev. May-Jun 2021;45(3 Suppl 1):1-37.

12. https://www.ema.europa.eu/en/documents/product-information/kaftrio-epar-product-information_en.pdf

13. Zemanick ET, Taylor-Cousar JL, Davies J, et al. A Phase 3 Open-Label Study of Elexacaftor/Tezacaftor/Ivacaftor in Children 6 through 11 Years of Age with Cystic Fibrosis and at Least One F508del Allele. Am J Respir Crit Care Med. 2021 Jun 15;203(12):15221532.

14. https://www.aifa.gov.it/web/guest/fondo-nazionale-aifa 